\title{
Utilização de contrairritante no tratamento da fixação dorsal de patela intermitente em equinos: relato de casos
}

[The use of counterirritant in the treatment of upward fixation of intermittent patella in horses: case report]

\author{
M.J. Watanabe ${ }^{1}$, M.D. Pyles ${ }^{2}$, J.M. Alonso ${ }^{1}$, D.Q. França ${ }^{1}$, A. L.M. Yamada ${ }^{1}$, \\ C.A. Hussni ${ }^{1}$, A.L.G. Alves ${ }^{1}$ \\ ${ }^{1}$ Faculdade de Medicina Veterinária e Zootecnia - UNESP - Botucatu, SP \\ ${ }^{2}$ Médico veterinário autônomo - Botucatu, SP
}

\begin{abstract}
RESUMO
Em nove equinos atendidos por apresentarem fixação dorsal de patela intermitente, optou-se pela aplicação de $2 \mathrm{~mL}$ de contrairritante na região subcutânea, ao longo dos ligamentos patelares medial e intermédio. No período de 12 a 48h após a aplicação, os animais apresentaram aumento de volume e de temperatura local, sensibilidade dolorosa à palpação e relutância à locomoção. Após esse período, os sinais clínicos de inflamação e fixação dorsal de patela foram gradativamente diminuindo até o sétimo dia, em sete dos nove animais avaliados. Após a remissão dos sinais de inflamação, dois animais não responderam ao tratamento, sendo necessária a repetição em um dos casos e realização de desmotomia patelar medial no outro. A aplicação de contrairritante foi eficaz na remissão do sinal clínico de fixação dorsal da patela intermitente. Comparativamente às técnicas cirúrgicas para a correção da enfermidade descritas na literatura, o tratamento promoveu melhora precoce dos sinais, curto período de convalescência e praticidade na realização.
\end{abstract}

Palavras-chave: fixação de patela, ligamentos patelares, tratamento conservativo, contrairritante

\begin{abstract}
Nine horses were admitted showing intermittent lameness due to upward fixation of the patella (UFP) and were injected with $2 \mathrm{~mL}$ of counter-irritant subcutaneously, through the middle and medial patellar ligaments. From 12 to 48 hours after application, these animals showed increased volume and local temperature, painful response to palpation and reluctance to bear weight. Subsequently, the inflammatory signs were gradually decreasing until the seventh day, along with signs of UFP in seven of the nine animals. After remission of inflammatory signs, two of the nine horses did not respond to the treatment. In these two animals, one horse needed a repeated counter-irritant injection and the other received the medial patellar desmotomy. The use of counter-irritant was effective in the remission of clinical signs of UFP. In comparison with the surgical techniques described in literature used to correct this disease, the proposed treatment promoted early improvement of clinical signs, short term recovery and was easily performed.
\end{abstract}

Keywords: conservative treatment, patellar attachment, patellar ligaments, revulsive

\section{INTRODUÇÃO}

A articulação femorotibiopatelar, considerada a maior e mais elaborada de todas as articulações, estrutura-se na junção de duas articulações, a femoropatelar e a femoro-tibial (Sisson, 1986). A estabilização articular é realizada por 14

Recebido em 20 de outubro de 2011

Aceito em 26 de novembro de 2012

E-mail: watanabe@fmvz.unesp.br ligamentos, sendo dois femoropatelares, três patelares, dois colaterais, dois cruzados, dois tibiais craniais, dois tibiais caudais e um ligamento menisco femorocaudal (Martins et al., 2007).

Os principais movimentos dessa articulação são os de flexão e extensão. Na posição de apoio do 
membro, o ângulo caudal é de aproximadamente $150^{\circ}$ e, quando em movimento, a extensão é incompleta, ou seja, os eixos longitudinais do fêmur e da tíbia não atingem $180^{\circ}$. A patela desliza-se sobre as trócleas do fêmur na região proximal durante a extensão e na distal durante a flexão (Sisson, 1986), sendo essa movimentação relacionada diretamente ao grupo muscular quadríceps femoral e aos ligamentos patelares. O grupo quadríceps femoral se insere na porção proximal da patela e promove o desencaixe da patela da crista troclear medial, mantendo a patela alinhada dentro do sulco troclear durante a locomoção (Martins et al., 2007). Os ligamentos patelares (lateral, intermédio e medial) se localizam peri e extra-articular, entre os coxins gordurosos e a cápsula articular, e atuam como inserções do quadríceps femoral, ligando patela à tíbia (Dyson, 2002).

A fixação dorsal de patela (FDP) ocorre quando não há o movimento de desencaixe da patela no sulco troclear medial do fêmur, resultando, assim, na impossibilidade de flexão da articulação femorotibial e, em virtude do aparato recíproco, durante a locomoção, o membro pélvico mantém-se em extensão estando as articulações distais flexionadas (Tnibar, 2003). Essa afecção foi descrita em bovinos, alpacas e búfalos, clinicamente caracterizada pela impossibilidade de flexão do membro, com hiperextensão de um ou de ambos os membros pélvicos em posição abduzida caudalmente (Sullins, 2002).

A FDP está entre as afecções mais importantes dessa articulação, sendo responsável por aproximadamente $15 \%$ das claudicações resultantes de afecções de tecidos moles que acometem os membros pélvicos (Jeffcott, 1982; Martins, et al., 2006). Os tratamentos preconizados dividem-se em conservadores e cirúrgicos, decisão esta relacionada com a severidade da enfermidade (Tnibar, 2003). Em relação aos conservadores, podem-se utilizar programas de condicionamento físico, aplicação de ferraduras com elevação dos talões (Tnibar, 2003) e aplicação de substâncias contrairritantes na região dos ligamentos patelares (Sullins, 2002). Já em se tratando dos tratamentos cirúrgicos, citam-se o splitting ligamentar e a desmotomia patelar medial, sendo esta última a técnica mais frequentemente realizada, mesmo sendo recomendada apenas em casos severos e quando não há resposta aos demais tratamentos (Tnibar, 2002), pois foram observadas complicações decorrentes da perda da função do ligamento patelar medial (Tnibar, 2002).

Neste sentido, com o presente relato, objetivouse descrever resultados clínicos da aplicação de contrairritante como método para tratamento conservativo de cavalos com fixação dorsal de patela intermitente.

\section{CASUÍSTICA}

Foram atendidos nove equinos que apresentavam quadro característico de FDP intermitente em graus variados de severidade. Destes, oito eram animais de até três anos de idade, em início de treinamento, e um com 10 anos de idade. Seis animais eram da raça Quarto-de-Milha, um da Brasileiro de Hipismo, um da Mangalarga Paulista e um sem raça definida.

Após o diagnóstico da afecção, o procedimento realizado consistiu de tricotomia e antissepsia da região dos ligamentos patelares, sedação com $0,5 \mathrm{mg} / \mathrm{kg}$ de cloridrato de xilazina a $10 \%$ (Sedazine - Fort Dodge - Brasil) pela via intravenosa e aplicação do contrairritante à base de cloreto de mercúrio, cânfora, álcool isopropílico, iodo, iodeto de potássio e azeite de terebintina (Workalin ${ }^{\circledR}$ injetável - Agropharma - Argentina), do qual se injetaram $2 \mathrm{~mL}$ na região subcutânea, no sentido longitudinal dos ligamentos patelares medial e intermédio. Nos dias seguintes ao tratamento, os animais foram mantidos em repouso em piquetes ou baias, e não foram administrados antibióticos e antiinflamatórios.

A afecção apresentou-se bilateral em sete dos nove animais, semelhante ao reportado na literatura (Tnibar, 2002). Assim, todos os animais foram submetidos à aplicação nos membros pélvicos, sendo o produto aplicado de maneira similar em ambos os membros. Também, todos os animais tratados foram considerados com fixação dorsal de patela intermitente e de grau moderado a grave, uma vez que, durante a locomoção em linha reta, nos casos moderados, houve fixação intermitente da patela, podendo ser audível o som de reposicionamento da patela e, nos casos graves, observou-se impossibilidade repetida de extensão da região distal do membro (Tnibar, 2002). 
Nesses casos, a claudicação foi caracterizada como restritiva mecânica, porém, mesmo não sendo notadas alterações articulares no presente relato, em casos crônicos podem se observar distensão da articulação femoropatelar e sinovite (Bennet et al., 1977; Clegg e Butson, 1996; Sullins, 2002).

Entre os tratamentos conservadores que poderiam ter sido empregados, citam-se a aplicação de ferraduras com elevação dos talões, a fim de se limitar a extensão da articulação femorotibial e a adoção de programas de condicionamento físico visando ao fortalecimento da musculatura do quadríceps e à melhora da coordenação entre os músculos flexores e extensores que agem nessa articulação (Tnibar, 2003). Porém, esses são recomendados para casos de grau leve de fixação dorsal de patela.

Optou-se pela aplicação de contrairritante, pois dos nove animais apenas um era utilizado para lazer, sendo que os outros oito tinham como principal função a atividade atlética, levando-se em consideração os estudos que relacionam alterações morfofuncionais decorrentes da desmotomia patelar medial. Gibson et al. (1989) avaliaram 12 equinos hígidos submetidos à desmotomia patelar medial unilateral e observaram fragmentação do aspecto distal da patela e proliferação óssea próxima à inserção do ligamento patelar intermédio em 11 destes. McIlwraith (1990), Jansson (1996), Stick e Nickels (2006) também relataram a ocorrência de fragmentação osteocondral da patela, claudicação e rigidez de moderada à severa da articulação femorotibiopatelar. Wright e Rose (1989) e Riley e Yovich (1991) verificaram fraturas de patela como complicação da desmotomia patelar medial. Baccarin et al. (2009) observaram instabilidade patelar por meio de exame radiográfico que demonstrou aumento do ângulo entre a superfície articular proximal da patela e a superfície cranial distal do fêmur. Martins et al. (2006) realizaram controle ultrassonográfico e observaram como complicações: desmite patelar intermédia, entesiófitos, efusão femoropatelar e do recesso articular femoral medial.

Em todos os animais, a técnica de aplicação do contrairritante foi similar à descrita por Sullins (2002), sendo recomendada a aplicação pela via subcutânea na região dos ligamentos patelares medial e intermédio, com o animal em apoio quadrupedal e sob sedação. No presente relato, os animais também foram submetidos à aplicação sob sedação com agonista alfa-2 adrenérgico, sem a necessidade de infiltração anestésica local, o que possibilitou a aplicação do fármaco sobre os ligamentos, tendo o cuidado para a agulha não transpassar o diâmetro do ligamento e acidentalmente administrar-se o fármaco na articulação femorotibial, uma vez que existe contato dos ligamentos com a membrana sinovial (Brown et al., 1984).

No período de 12 a 48h após a aplicação, os animais apresentaram aumento de volume e temperatura local, sensibilidade dolorosa à palpação e relutância à locomoção, devido à intensa inflamação provocada pelo contrairritante, similarmente ao descrito por Sullins (2002). Após esse período, os sinais de inflamação e fixação dorsal de patela foram gradativamente diminuindo até o sétimo dia, em sete dos nove animais (Fig. 1). Similarmente, Tnibar (2002) observou, em equinos submetidos à cirurgia de splitting, aumento de volume e calor moderado, com sensibilidade dolorosa variável à palpação no local da cirurgia. A remissão bilateral da FDP foi observada em três dos sete pacientes após $24 \mathrm{~h}$ da cirurgia, enquanto os outros tiveram resolução em até 12 dias.

Em nenhum dos animais foram aplicados anti-inflamatórios após a terapia, similar aos tratamentos propostos por Brown et al. (1984) e Tnibar (2002), e diferente dos animais submetidos à desmotomia patelar medial, uma vez que o processo inflamatório seria um componente importante no efeito sobre a resolução da enfermidade (Tnibar, 2003).

Após a remissão dos sinais de inflamação, dois cavalos não responderam ao tratamento, sendo necessária a repetição do tratamento em um dos casos e realização de desmotomia patelar medial no outro. Como suspeita da não resposta à primeira aplicação destes, sugere-se a influência da técnica utilizada que ainda não está estabelecida quanto ao volume e ao modo a ser aplicado, ressaltando que a utilização de contrairritante nos ligamentos patelares ainda foi pouco descrita em casos clínicos de FDP (Brown et al., 1984; Van Hoogmoed et al., 2002). 

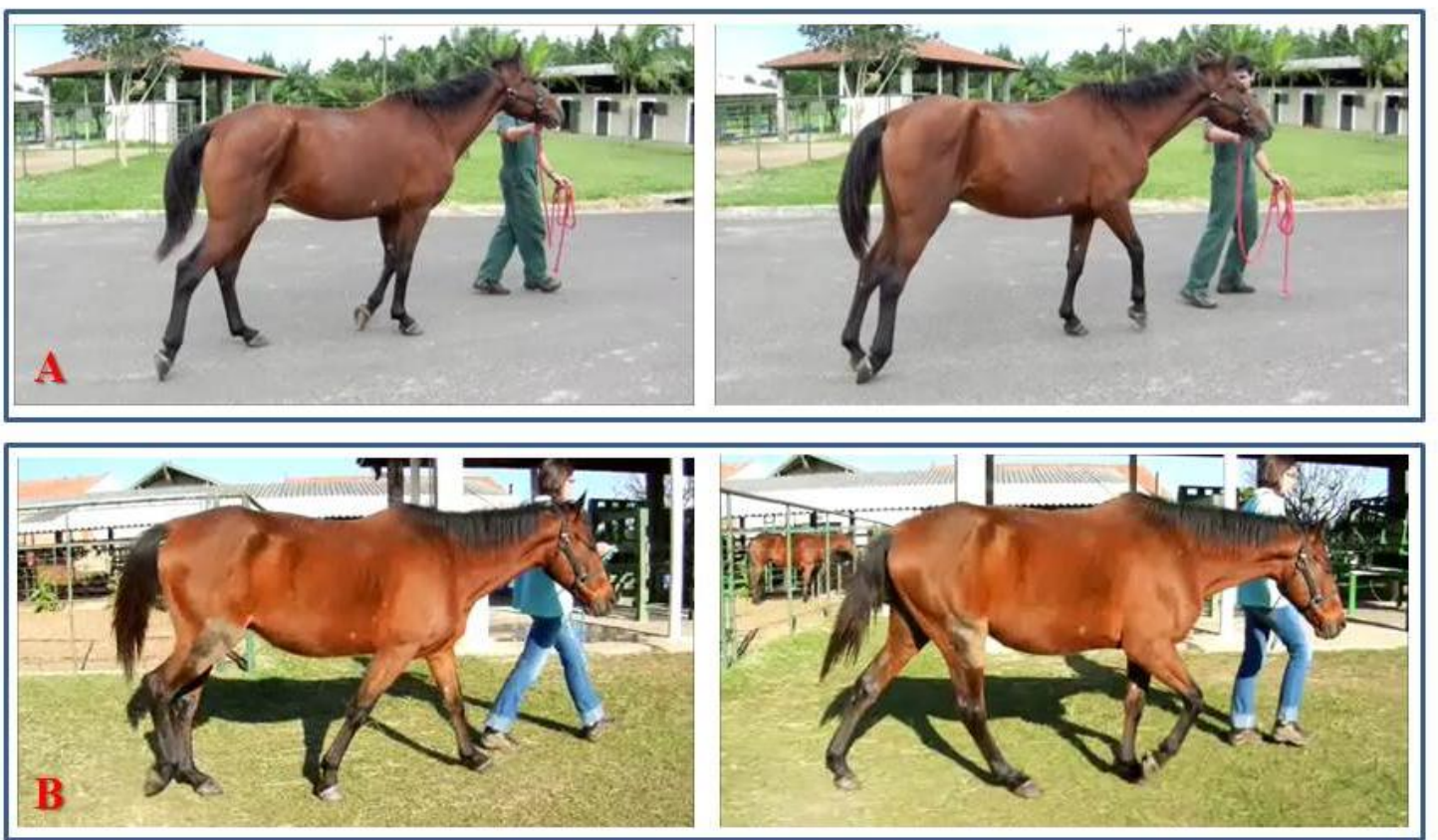

Figura 1.A. Impossibilidade de flexão do membro, com posição abduzida caudalmente do membro pélvico direito, em cavalo com fixação dorsal de patela. B - Ausência dos sinais de fixação dorsal de patela. Controle clínico 50 dias após a aplicação do contrairritante.

Assim, o presente trabalho corrobora o de Tnibar (2003), o qual sugere, como mecanismo de ação do contrairritante, o efeito inflamatório, que causa modificações das dimensões (espessamento e encurtamento) e do remodelamento ligamentar. Brown et al. (1984) realizaram a aplicação de solução contrairritante nos ligamentos patelares de 10 pôneis hígidos e observaram, ao exame histológico, reação inflamatória intensa e, também, ruptura das fibras colágenas, com produção de tecido fibroso e aumento do diâmetro dos ligamentos. Igualmente, Van Hoogmoed et al. (2002) demonstraram a eficácia desses agentes no desenvolvimento de inflamação e fibroplasia dos ligamentos patelares medial e intermédio por meio de avaliação ultrassonográfica e histológica. O tratamento cirúrgico splitting, descrito por Tnibar (2002) e realizado em equinos com sinais de FDP bilateral, possui mecanismo de ação similar. Os sinais de desmite do terço proximal do ligamento patelar medial foram observados nos animais imediatamente após a realização da técnica. A melhora clínica dos animais submetidos a tal procedimento foi atribuída também ao aumento do diâmetro ligamentar na região submetida ao splitting. Porém, esse procedimento seria indicado nos casos de não resposta ao tratamento conservador, pois a técnica necessita de anestesia geral e deve ser guiada por ultrassonografia, o que limita a utilização do procedimento em condições de campo.

A aplicação de contrairritante foi eficaz na remissão do sinal clínico de fixação dorsal da patela intermitente. Comparativamente às técnicas cirúrgicas descritas na literatura para a correção da enfermidade, o tratamento promoveu melhora precoce dos sinais, curto período de convalescência e praticidade na realização. 


\section{REFERÊNCIAS}

BACCARIN, R.Y.; MARTINS, E.A.; HAGEN, S.C. Patellar instability following experimental medial patellar desmotomy in horses. Vet. Comp. Orthop. Traumatol., v.22, p.27-31. 2009.

BENNETT, D.; CAMPBELL, J.R; RAWLINSON, J.R. Coxofemoral luxation complicated by upward fixation of the patella in the pony. Equine Vet. J., v.9, p.192-4, 1977.

BROWN, M.P.; MOON, P.D.; BUERGELT, C.D. The effects of injection of an iodine counterirritant into the patellar ligaments of ponies: Application to stifle lameness. J. Equine Vet. Sci, v.4, p.82-87, 1984.

CLEGG, P.D.; BUTSON, R.J. Treatment of coxofemoral luxation secondary to upward fixation of patella in Shetland pony. Vet. Rec. v.138, p.134-137, 1996.

DYSON, S.J. Normal ultrasonographic anatomy and injury of the patellar ligaments in the horse. Equine Vet. J., v.34, p.258-264, 2002.

GIBSON, K.T.; McILWRAITH, C.W. et al. Production of patellar lesions by medial patellar desmotomy in normal horses. Vet. Surg., v.18, p.466-471, 1989.

JANSSON, N. Treatment for upward fixation of the patella in the horse by medial patellar desmotomy: indications and complications. Equine Pract., v.18, p.24-29, 1996.

JEFFCOTT, L.B.; KOLD, S.E. Stifle lameness in the horse: a survey of 86 referred cases. Equine Vet. J., v.14, p.31-39, 1982.

MARTINS, E.A.N.; SILVA, L.C.L.C.; BACCARIN, R.Y.A. Ultrasonographic changes of the equine stifle following experimental medial patellar desmotomy. Can. Vet. J., v.47, p.471-474, 2006.

MARTINS, E.A.N.; SILVA， L.C.L.C.; BACCARIN, R.Y.A. Líquido sinovial da articulação femuropatelar após desmotomia patelar medial experimental em equinos. Cienc. Rural, v.37, p. 784-788, 2007.
McILWRAITH, C.W. Osteochondral fragmentation of the distal aspect of the patella in horses. Equine Vet. J., v.22, p.157-163, 1990.

RILEY, C.B.; YOVICH, J.V. Fracture of the apex of the patella after medial patellar desmotomy in a horse. Aust. Vet. J., v.68, p.3739, 1991.

SISSON, S. Articulações. In: GETTY, R. (5.ed) Anatomia dos animais domésticos, p. 338-344, 1986.

STICK, J.A.; NICKELS, F.A. Stifle. In: AUER \& STICK. Equine Surgery, 3ed., 2006. p.13251326.

SULLINS, K.E. Parte XII - Soldra. In: STASHAK, T.S. (5.ed.) Claudicação em equinos Segundo Adams. p.961-962, 2002.

TNIBAR, M.A. Medial patellar ligament splitting for the treatment of upward fixation of the patella in 7 equids. Vet. Surg., v.31, p.462467, 2002.

TNIBAR, M.A. Treatment of upward fixation of the patella in the horse: an update. Equine Vet. Educ., v.15, p.236-242, 2003.

VAN HOOGMOED, L.M.; AGNEW, D.W.; WHITCOMB, M. Ultrasonographic and histologic evaluation of medial and middle patellar ligaments in exercised horses following injection with ethanolamine oleate and $2 \%$ iodine in almond oil. Am. J. Vet. Res., v.63, p.738-743, 2002.

WRIGHT, J.D.; ROSE, R.J. Fracture of the patella as a possible complication of medial patellar desmotomy. Aus.Vet. J., v.66, p.189-190, 1989. 